\title{
Selecting the best stochastic systems for large scale engineering problems
}

\author{
Mahmoud H. Alrefaei ${ }^{1}$, Mohammad H. Almomani ${ }^{2}$, Sarah N. Alabed Alhadi ${ }^{3}$ \\ ${ }^{1,3}$ Department of Mathematics and Statistics, Jordan University of Science and Technology, Jordan \\ ${ }^{2}$ Department of Mathematics, The Hashemite University, Jordan
}

\begin{abstract}
Article Info
Article history:

Received Jul 21, 2020

Revised Jan 5, 2021

Accepted Jan 13, 2021

\section{Keywords:}

Asymptotic approximation Optimal computing budget allocation Ordinal optimization Probability of correct selection

ABSTRACT

Selecting a subset of the best solutions among large-scale problems is an important area of research. When the alternative solutions are stochastic in nature, then it puts more burden on the problem. The objective of this paper is to select a set that is likely to contain the actual best solutions with high probability. If the selected set contains all the best solutions, then the selection is denoted as correct selection. We are interested in maximizing the probability of this selection; $\mathrm{P}(\mathrm{CS})$. In many cases, the available computation budget for simulating the solution set in order to maximize $\mathrm{P}(\mathrm{CS})$ is limited. Therefore, instead of distributing these computational efforts equally likely among the alternatives, the optimal computing budget allocation (OCBA) procedure came to put more effort on the solutions that have more impact on the selected set. In this paper, we derive formulas of how to distribute the available budget asymptotically to find the approximation of $\mathrm{P}(\mathrm{CS})$. We then present a procedure that uses OCBA with the ordinal optimization (OO) in order to select the set of best solutions. The properties and performance of the proposed procedure are illustrated through a numerical example. Overall results indicate that the procedure is able to select a subset of the best systems with high probability of correct selection using small number of simulation samples under different parameter settings.
\end{abstract}

This is an open access article under the CC BY-SA license.

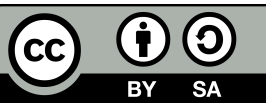

\section{Corresponding Author:}

Mahmoud H. Alrefaei

Department of Mathematics and Statistics

University of Science and Technology

Jordan

Email: alrefaei@just.edu.jo

\section{INTRODUCTION}

Consider a stochastic optimization problem at which it is sought to select a set of best solutions with high probability, when the solution set is very large. For the presence of noise, one needs to estimate the objective function values of all or sometimes some of the solutions. Simulation is the primary way to get these estimates, however, simulation requires a huge amount of computational time, especially when the set of alternative systems is large. Therefore, getting a precise estimate of each alternative could be a time consuming, and much time will be spent on alternatives that are not likely to be optimal. The optimal computing budget allocation (OCBA) proposed by Chen et al. [1] that puts more effort of computation on the solutions that have more 2 impact on the selection while not neglecting the other solutions. Recently, the OCBA has been studied extensively in the literature, Xiao et al. [2] studied simulation optimization using genetic algorithms with OCBA, Zhang et al. [3] have considered the OCBA for selecting an optimal subset, Xiao et al. [4] have 
considered the OCBA for ranking the top designs with stochastic constraints and Zhang et al. [5] have studied the OCBA for particle swarm optimization in stochastic optimization. Zhu et al. [6] have combined the OCBA with the time dilation (TD), which is a single-run simulation optimization algorithm to solve an optimization problem and implement it to solve remote diagnostic (RD) problem in the semiconductor sector.

One of the methods that is commonly used to solve the large size stochastic optimization problem is the particle swarm optimization (PSO), which is a random search method that iteratively arrange the particles around the state space according to simple mathematical formulae over the particle's position and velocity [7]. See also [8] for a PSO and simulated annealing (SA) algorithm. Manusov et al. [9] have used the PSO to find the optimal distribution and powers of reactive power compensation units. Yoganandini and Anitha [10] have used the PSO to maximize the power point tracking (MPPT) in order to enhance the efficiency of the photo voltaic (PV) systems. More methods used to solve optimization methods especially for solving optimal power flow can be found in Abd Rahman et al. [11].

The OCBA has been implemented in many applications, for instance Liu [12] has applied the OCBA to solve the $N-k$ problem that asks whether there exists a set of $k$ or fewer elements out of $N$ elements whose removal would cause the system to fail in a power grid generation model Cao et al. [13] have implemented the OCBA to find the optimal maintenance policies for the system to improve system availability so as to increase profit margin.

In many cases when the size of the problem is very large, then one can relax the problem to locate a good enough optimal set rather than estimating each alternative precisely, this is the essence of the ordinal optimization (OO) proposed by Ho et al. [14]. The idea of OO is to select a random subset from the solution set, then use any known optimization method to select the best system in the smaller set if the objective to select one best solution. Chen [15] has proposed a procedure using the idea of $O O$ with ranking and selection methods to improve the performance of indifference zone $(I Z)$ selection procedure.

In this paper, we propose a method for selecting a set that contains the best solutions among large but finite set of stochastic solutions. We first derive new asymptotic formulas for the number of samples that can be allocated to each solution, in order to maximize the probability of correct selection. Of course, there are formulas in the literature, however, the proposed formulas allow us to approximate the probability of correct selection $P(C S)$. The proposed method is implemented in two examples. It can be used also to solve large scale practical problems such as the optimal distribution and powers of reactive power compensation units Manusov et al. [9], maximize the power point tracking (MPPT) in a photo voltaic (PV) systems Yoganandini and Anitha [10], configuration of multi-agent system Hasan and Rafaat [16] and optimal design of digital and analog filters [17].

Most of the previous work use combined procedures to select the best or a set of best solution systems, when the number of solutions is large. For example, Gao et al. [18] proposed a sequential budget allocation framework for simulation optimization. Alrefaei and Alawneh [19] have used two stage approach to select the best simulated system among relatively large number of systems. Almomani and Abdul Rahman [20] have proposed a sequential selection approaches to select a good system when the number of alternatives is large. Almomani and Alrefaei [21] proposed a sequential algorithm for selecting a subset that contains good enough systems when the number of alternatives is very large. For more sequential procedures, see [22-25].

The paper is organized as follows: In section 2, we formulate the problem, in section 2.1, we present a derivation of the number of samples needed in $O C B A_{m}$ to maximize $\mathrm{P}(\mathrm{CS})$, in this section we also present the approximation of $\mathrm{P}(\mathrm{CS})$ and propose the procedure for selecting the best $\mathrm{m}$ systems. In section 3 . we implement the proposed procedure for solving an inventory problem and discuss the results. Finally, concluding remarks are presented in section 4.

\section{RESEARCH METHOD}

Let $S_{m}$ be the set of the best solution of $k$ solution systems. When the available computational budget $T$ is known, we seek to distribute this budget to all alternative solutions by allocating $N_{i}$ samples for each solution $i$ in order to maximize the $P(C S)$. Therefore, the problem can be formulated as (1).

$$
\begin{array}{r}
\max _{N_{1}, \ldots, N_{k}} P\left(C S_{m}\right) \\
\text { s.t. } \sum_{i=1}^{k} N_{i}=T
\end{array}
$$


Assume that $Y_{i j}$ is the $j^{\text {th }}$ simulation sample of $Y_{i}, j=1, \ldots, N_{i}$ and $\overline{Y_{i}}=1 / N_{i} \sum_{j=1}^{N_{i}} Y_{i j}$ is the estimated mean. Using the methods of multiple replications method of simulation guarantees that $\overline{Y_{i}}$ is considered as asymptotically normally distributed with mean $Y_{i}$ and variance $\sigma_{i}^{2} / N_{i}$. When $\sigma_{i}^{2}$ is unknown, it can be estimated by $s_{i}^{2}$ for $Y_{i}$.

In order to select the set $S_{m}$ that contains the $m$ systems with the smallest means, let $\bar{Y}_{i_{r}}$ be the $r$-th smallest (order statistic) of $\left\{\bar{Y}_{1}, \bar{Y}_{2}, \ldots, \bar{Y}_{n}\right\}$, i.e. $\bar{Y}_{i_{1}} \leq \bar{Y}_{i_{2}} \leq \ldots \leq \bar{Y}_{i_{n}}$. Then, the set will be given by $S_{m}=\left\{i_{1}, i_{2}, \ldots, i_{m}\right\}$. The correct selection is that $S_{m}$ contains the actual $m$ smallest means, i.e. $C S_{m}=\left\{\max _{i \in S_{m}} Y_{i} \leq \min _{i \notin S_{m}} Y_{i}\right\}$, where $Y_{i}$ is the mean of system $i$.

\subsection{Optimal computing budget allocation for selecting the best $\mathbf{m}$}

Chen et al. [26])

Let $\hat{Y}_{i}$ be independent samples of $\bar{Y}_{i}$ obtained by simulation, then $P(C S)$ can be approximated as: (see by

$$
\begin{aligned}
P\left(C S_{m}\right) & =P\left\{\hat{Y}_{i} \leq \hat{Y}_{j}\right\}, \forall i \in S_{m}, j \notin S_{m} \\
& \geq P\left\{\hat{Y}_{i} \leq c \text { and } \hat{Y}_{j} \geq c\right\}, i \in S_{m}, j \notin S_{m} \\
& =\prod_{i \in S_{m}} P\left\{\hat{Y}_{i} \leq c\right\} \prod_{i \notin S_{m}} P\left\{\hat{Y}_{i} \geq c\right\}=A P C S_{m}
\end{aligned}
$$

where $c$ is a constant between $Y_{m}$ and $Y_{m+1}$.

Let $\alpha_{i}=\left(Y_{i}-c\right) / \sigma_{i}$ then for $i \in S_{m}, \alpha_{i}<0$. Since $\hat{Y}_{i}$ is normally distributed with mean $Y_{i}$ and variance $\sigma_{i}^{2} / N_{i}$, for large $N_{i}$, it can be shown by simple calculation, see (Ross [27] (chapter 5 section 3)) that:

$$
\begin{aligned}
P\left(\hat{Y}_{i} \leq c\right) & =P\left(\frac{\hat{Y}_{i}-Y_{i}}{\sigma_{i} / \sqrt{N_{i}}} \leq \frac{c-Y_{i}}{\sigma_{i} / \sqrt{N_{i}}}\right) \\
& =1-\left(1-P\left(\frac{\hat{Y}_{i}-Y_{i}}{\sigma_{i} / \sqrt{N_{i}}} \leq-\alpha_{i} \sqrt{N_{i}}\right)\right) \\
& >1+\frac{1}{\sqrt{2 \pi}} \frac{1}{\sqrt{N_{i}} \alpha_{i}} \exp \left(\frac{-\alpha_{i}^{2} N_{i}}{2}\right), \text { for large } N_{i}
\end{aligned}
$$

We approximate $P\left(\hat{Y}_{i} \leq c\right)$ by its lower bound, therefore

$$
P\left(\hat{Y}_{i} \leq c\right) \approx 1+\frac{1}{\sqrt{2 \pi}} \frac{1}{\sqrt{N_{i}} \alpha_{i}} \exp \left(\frac{-\alpha_{i}^{2} N_{i}}{2}\right)
$$

Similarly for $i \notin S_{m}$, then $\alpha_{i}>0$, therefore

$$
\begin{aligned}
P\left(\hat{Y}_{i} \geq c\right) & =P\left(\frac{\hat{Y}_{i}-Y_{i}}{\sigma_{i} / \sqrt{N_{i}}} \geq \frac{c-Y_{i}}{\sigma_{i} / \sqrt{N_{i}}}\right) \\
& =P\left(\frac{\hat{Y}_{i}-Y_{i}}{\sigma_{i} / \sqrt{N_{i}}} \leq \frac{Y_{i}-c}{\sigma_{i} / \sqrt{N_{i}}}\right) \\
& =1-\left(1-P\left(\frac{\hat{Y}_{i}-Y i}{\sigma_{i} / \sqrt{N_{i}}} \leq \frac{Y_{i}-c}{\sigma_{i} / \sqrt{N_{i}}}\right)\right) \\
& >1-\frac{1}{\sqrt{2 \pi}} \frac{1}{\sqrt{N_{i}} \alpha_{i}} \exp \left(\frac{-\alpha_{i}^{2} N_{i}}{2}\right), \text { for large } N_{i}
\end{aligned}
$$

again we let

$$
P\left(\hat{Y}_{i} \geq c\right) \approx 1-\frac{1}{\sqrt{2 \pi}} \frac{1}{\sqrt{N_{i}} \alpha_{i}} \exp \left(\frac{-\alpha_{i}^{2} N_{i}}{2}\right)
$$

Now we provide asymptotic analysis of the probability of correct selection 


$$
\begin{aligned}
A P C S_{m} & =\prod_{i \in S_{m}} P\left\{\hat{Y}_{i} \leq c\right\} \prod_{j \neq S_{m}} P\left\{\hat{Y}_{j} \geq c\right\} \\
& \approx \prod_{i \in S_{m}}\left[1+\frac{1}{\sqrt{2 \pi}} \frac{1}{\sqrt{N_{i}} \alpha_{i}} \exp \left(\frac{-\alpha_{i}^{2} N_{i}}{2}\right)\right] \prod_{i \notin S_{m}}\left[1-\frac{1}{\sqrt{2 \pi}} \frac{1}{\sqrt{N_{i}} \alpha_{i}} \exp \left(\frac{-\alpha_{i}^{2} N_{i}}{2}\right)\right] \\
& =E A P C S_{m}
\end{aligned}
$$

where EAPCS denotes the estimated approximate probability of correct selection, note that $\alpha_{i}<0$ for $i \in S_{m}$ and $\alpha_{i}>0$ for $i \notin S_{m}$.

The optimization problem (1) becomes,

$$
\begin{aligned}
\max _{N_{1}, N_{2}, \ldots, N_{k}} & \prod_{i \in S_{m}}\left[1+\frac{1}{\sqrt{2 \pi}} \frac{1}{\sqrt{N_{i}} \alpha_{i}} \exp \left(\frac{-\alpha_{i}^{2} N_{i}}{2}\right)\right] \prod_{i \notin S_{m}}\left[1-\frac{1}{\sqrt{2 \pi}} \frac{1}{\sqrt{N_{i}} \alpha_{i}} \exp \left(\frac{-\alpha_{i}^{2} N_{i}}{2}\right)\right] \\
\text { subject to } & \sum_{i=1}^{k} N_{i}=T \\
& N_{i} \in \mathbb{N}, \quad i=1,2, \ldots, k
\end{aligned}
$$

Let $F$ be the Lagrangian relaxation function of the optimization problem (5) then,

$$
\begin{aligned}
F= & \prod_{i \in S_{m}}\left[1+\frac{1}{\sqrt{2 \pi}} \frac{1}{\sqrt{N_{i}} \alpha_{i}} \exp \left(\frac{-\alpha_{i}^{2} N_{i}}{2}\right)\right] \prod_{i \notin S_{m}}\left[1-\frac{1}{\sqrt{2 \pi}} \frac{1}{\sqrt{N_{i}} \alpha_{i}} \exp \left(\frac{-\alpha_{i}^{2} N_{i}}{2}\right)\right] \\
& -\lambda \sum_{i=1}^{k}\left(N_{i}-T\right)
\end{aligned}
$$

Take the partial derivatives $\frac{\partial F}{\partial N_{i}}$ for the lagrangian function $F$, then for $i \in S_{m}$ we get,

$$
\begin{aligned}
\frac{\partial F}{\partial N_{i}}=\prod_{j \in S_{m} \neq i}[1+ & \left.\frac{1}{\sqrt{2 \pi}} \frac{1}{\sqrt{N_{j}} \alpha_{j}} \exp \left(\frac{-\alpha_{i}^{2} N_{i}}{2}\right)\right] \prod_{j \notin S_{m}}\left[1-\frac{1}{\sqrt{2 \pi}} \frac{1}{\sqrt{N_{j}} \alpha_{j}} \exp \left(\frac{-\alpha_{i}^{2} N_{i}}{2}\right)\right] \\
& \times\left[\frac{-1}{2 \sqrt{2 \pi}} \frac{1}{N_{i} \sqrt{N_{i}} \alpha_{i}} \exp \left(\frac{-\alpha_{i}^{2} N_{i}}{2}\right)-\frac{1}{\sqrt{2 \pi}} \frac{\alpha_{i}}{2 \sqrt{N_{i}}} \exp \left(\frac{-\alpha_{i}^{2} N_{i}}{2}\right)\right]-\lambda
\end{aligned}
$$

Note that as $N_{i} \rightarrow \infty$, the second term in

$$
\frac{-1}{2 \sqrt{2 \pi}} \frac{1}{N_{i} \sqrt{N_{i}} \alpha_{i}} \exp \left(\frac{-\alpha_{i}^{2} N_{i}}{2}\right)-\frac{1}{\sqrt{2 \pi}} \frac{\alpha_{i}}{2 \sqrt{N_{i}}} \exp \left(\frac{-\alpha_{i}^{2} N_{i}}{2}\right)
$$

dominate the first term, so $\frac{\partial F}{\partial N_{i}}$ becomes

$$
\begin{aligned}
\frac{\partial F}{\partial N_{i}}= & \prod_{j \in S_{m} j \neq i}\left[1+\frac{1}{\sqrt{2 \pi}} \frac{1}{\sqrt{N_{j}} \alpha_{j}} \exp \left(\frac{-\alpha_{i}^{2} N_{i}}{2}\right)\right] \prod_{j \notin S_{m}}\left[1-\frac{1}{\sqrt{2 \pi}} \frac{1}{\sqrt{N_{j}} \alpha_{j}} \exp \left(\frac{-\alpha_{i}^{2} N_{i}}{2}\right)\right] \\
& \times\left[\frac{-1}{\sqrt{2 \pi}} \frac{\alpha_{i}}{2 \sqrt{N_{i}}} \exp \left(\frac{-\alpha_{i}^{2} N_{i}}{2}\right)\right]-\lambda
\end{aligned}
$$

For $i \notin S_{m}$ we get,

$$
\begin{aligned}
\frac{\partial F}{\partial N_{i}}=\prod_{j \in S_{m}}\left[1+\frac{1}{\sqrt{2 \pi}}\right. & \left.\frac{1}{\sqrt{N_{j}} \alpha_{j}} \exp \left(\frac{-\alpha_{j}^{2} N_{j}}{2}\right)\right] \prod_{j \notin S_{m} j \neq i}\left[1-\frac{1}{\sqrt{2 \pi}} \frac{1}{\sqrt{N_{j}} \alpha_{j}} \exp \left(\frac{-\alpha_{j}^{2} N_{j}}{2}\right)\right] \\
\times & {\left[\frac{1}{2 \sqrt{2 \pi}} \frac{1}{N_{i} \sqrt{N_{i} \alpha_{i}}} \exp \left(\frac{-\alpha_{i}^{2} N_{i}}{2}\right)+\frac{1}{\sqrt{2 \pi}} \frac{\alpha_{i}}{2 \sqrt{N_{i}}} \exp \left(\frac{-\alpha_{i}^{2} N_{i}}{2}\right)\right]-\lambda }
\end{aligned}
$$


Also the second term of

$$
\frac{1}{2 \sqrt{2 \pi}} \frac{1}{N_{i} \sqrt{N_{i}} \alpha_{i}} \exp \left(\frac{-\alpha_{i}^{2} N_{i}}{2}\right)+\frac{1}{\sqrt{2 \pi}} \frac{\alpha_{i}}{2 \sqrt{N_{i}}} \exp \left(\frac{-\alpha_{i}^{2} N_{i}}{2}\right)
$$

dominates the first term, therefore $\frac{\partial F}{\partial N_{i}}$ becomes

$$
\begin{aligned}
\frac{\partial F}{\partial N_{i}}= & \prod_{j \in S_{m}}\left[1+\frac{1}{\sqrt{2 \pi}} \frac{1}{\sqrt{N_{j}} \alpha_{j}} \exp \left(\frac{-\alpha_{j}^{2} N_{j}}{2}\right)\right] \prod_{j \notin S_{m} j \neq i}\left[1-\frac{1}{\sqrt{2 \pi}} \frac{1}{\sqrt{N_{j}} \alpha_{j}} \exp \left(\frac{-\alpha_{j}^{2} N_{j}}{2}\right)\right] \\
& \times\left[\frac{1}{\sqrt{2 \pi}} \frac{\alpha_{i}}{2 \sqrt{N_{i}}} \exp \left(\frac{-\alpha_{i}^{2} N_{i}}{2}\right)\right]-\lambda
\end{aligned}
$$

and $\frac{\partial F}{\partial \lambda}$ yields

$$
\frac{\partial F}{\partial \lambda}=\sum_{i=1}^{k}\left(N_{i}-T\right)
$$
$\frac{\partial F}{\partial \lambda}=0$

Now we use the Karush-Kuhn-Tucker (KKT) optimality conditions by letting $\frac{\partial F}{\partial N_{i}}=0, \forall i=1, \ldots k$, and For $i \in S_{m}$

$$
\begin{aligned}
& \prod_{j \in S_{m} j \neq i}\left[1+\frac{1}{\sqrt{2 \pi}} \frac{1}{\sqrt{N_{j}} \alpha_{j}} \exp \left(\frac{-\alpha_{j}^{2} N_{j}}{2}\right)\right] \prod_{j \notin S_{m}}\left[1-\frac{1}{\sqrt{2 \pi}} \frac{1}{\sqrt{N_{j}} \alpha_{j}} \exp \left(\frac{-\alpha_{j}^{2} N_{j}}{2}\right)\right] \\
& \times\left[\frac{-1}{\sqrt{2 \pi}} \frac{\alpha_{i}}{2 \sqrt{N_{i}}} \exp \left(\frac{-\alpha_{i}^{2} N_{i}}{2}\right)\right]=\lambda
\end{aligned}
$$

For $i \notin S_{m}$

$$
\begin{aligned}
& \prod_{j \in S_{m}}\left[1+\frac{1}{\sqrt{2 \pi}} \frac{1}{\sqrt{N_{j}} \alpha_{j}} \exp \left(\frac{-\alpha_{j}^{2} N_{i} j}{2}\right)\right] \prod_{j \notin S_{m} j \neq i}\left[1-\frac{1}{\sqrt{2 \pi}} \frac{1}{\sqrt{N_{j}} \alpha_{j}} \exp \left(\frac{-\alpha_{j}^{2} N_{j}}{2}\right)\right] \\
& \times\left[\frac{1}{\sqrt{2 \pi}} \frac{\alpha_{i}}{2 \sqrt{N_{i}}} \exp \left(\frac{-\alpha_{i}^{2} N_{i}}{2}\right)\right]=\lambda
\end{aligned}
$$

and $\frac{\partial F}{\partial \lambda}=0$ is just the constraint $\sum_{i=1}^{k} N_{i}=T$.

To find a relation between $N_{i}$ and $N_{j}$ we take three cases:

- Case I: $i, j \in S_{m}$

By (10), we get

$$
\begin{aligned}
& \frac{-\alpha_{i}}{\sqrt{N_{i}}} \exp \left(\frac{-\alpha_{i}^{2} N_{i}}{2}\right)-\frac{1}{2 \pi} \frac{\alpha_{i}}{\alpha_{j} \sqrt{N_{i}} \sqrt{N_{j}}} \exp \left(\frac{-\alpha_{i}^{2} N_{i}}{2}-\frac{\alpha_{j}^{2} N_{j}}{2}\right)= \\
& \frac{-\alpha_{j}}{\sqrt{N_{j}}} \exp \left(\frac{-\alpha_{j}^{2} N_{j}}{2}\right)-\frac{1}{2 \pi} \frac{\alpha_{j}}{\alpha_{i} \sqrt{N_{i}} \sqrt{N_{j}}} \exp \left(\frac{-\alpha_{i}^{2} N_{i}}{2}-\frac{\alpha_{j}^{2} N_{j}}{2}\right)
\end{aligned}
$$

As $N_{i}, N_{j} \rightarrow \infty$, the first term in each side dominates the second term, therefore

$$
\frac{-\alpha_{i}}{\sqrt{N_{i}}} \exp \left(\frac{-\alpha_{i}^{2} N_{i}}{2}\right)=\frac{-\alpha_{j}}{\sqrt{N_{j}}} \exp \left(\frac{-\alpha_{j}^{2} N_{j}}{2}\right)
$$

Take the $\log$ for both sides, and note that $\alpha_{i}<0$ since $i \in S_{m}$, we get

$$
\log \frac{-\alpha_{i}}{\sqrt{N_{i}}}+\left(\frac{-\alpha_{i}^{2} N_{i}}{2}\right)=\log \frac{-\alpha_{j}}{\sqrt{N_{j}}}+\left(\frac{-\alpha_{j}^{2} N_{j}}{2}\right)
$$


$t_{j} T$

Since $\sum_{i=1}^{k} N_{i}=T$, we can assume that $N_{i}=t_{i} T$, where $0 \leq t_{i} \leq 1$ and $i=1, \ldots, k$. Let $N_{i}=t_{i} T, N_{j}=$ then by simple calculations, we get

$$
\frac{-1}{2 T} \log \frac{t_{i} T}{\alpha_{i}^{2}}+\left(\frac{-\alpha_{i}^{2} t_{i}}{2}\right)=\frac{-1}{2 T} \log \frac{t_{j} T}{\alpha_{j}^{2}}+\left(\frac{-\alpha_{j}^{2} t_{j}}{2}\right)
$$

Let $T \rightarrow \infty$ we get the relation

$$
t_{i} \alpha_{i}^{2}=t_{j} \alpha_{j}^{2}
$$

Multiply by $T$, and note that $t_{i} T=N_{i}$,

$$
N_{i} \alpha_{i}^{2}=N_{j} \alpha_{j}^{2}
$$

Recall that $\alpha_{i}=\left(Y_{i}-c\right) / \sigma_{i}$, if we let $\delta_{i}=Y_{i}-c$, then

$$
\frac{N_{i}}{\sigma_{i}^{2} / \delta_{i}^{2}}=\frac{N_{j}}{\sigma_{j}^{2} / \delta_{j}^{2}}
$$

- Case II: $i \in S_{m}, j \notin S_{m}$

By (10) and (11)

$$
\begin{aligned}
& \frac{-1}{\sqrt{2 \pi}} \frac{\alpha_{i}}{2 \sqrt{N_{i}}} \exp \left(\frac{-\alpha_{i}^{2} N_{i}}{2}\right)\left[1-\frac{1}{\sqrt{2 \pi}} \frac{1}{\sqrt{N_{j}} \alpha_{j}} \exp \left(\frac{-\alpha_{j}^{2} N_{j}}{2}\right)\right] \\
= & \frac{1}{\sqrt{2 \pi}} \frac{\alpha_{j}}{2 \sqrt{N_{j}}} \exp \left(\frac{-\alpha_{j}^{2} N_{j}}{2}\right)\left[1+\frac{1}{\sqrt{2 \pi}} \frac{1}{\sqrt{N_{i}} \alpha_{i}} \exp \left(\frac{-\alpha_{i}^{2} N_{i}}{2}\right)\right]
\end{aligned}
$$

so

$$
\begin{aligned}
& \frac{-1}{\sqrt{2 \pi}} \frac{\alpha_{i}}{2 \sqrt{N_{i}}} \exp \left(\frac{-\alpha_{i}^{2} N_{i}}{2}\right)+\frac{1}{4 \pi} \frac{\alpha_{i}}{\sqrt{N_{i} N_{j}} \alpha_{j}} \exp \left(\frac{-\alpha_{i}^{2} N_{i}}{2}\right) \exp \left(\frac{-\alpha_{j}^{2} N_{j}}{2}\right) \\
= & \frac{1}{\sqrt{2 \pi}} \frac{\alpha_{j}}{2 \sqrt{N_{j}}} \exp \left(\frac{-\alpha_{j}^{2} N_{j}}{2}\right)+\frac{1}{4 \pi} \frac{\alpha_{j}}{\sqrt{N_{i} N_{j}} \alpha_{i}} \exp \left(\frac{-\alpha_{j}^{2} N_{j}}{2}\right) \exp \left(\frac{-\alpha_{i}^{2} N_{i}}{2}\right)
\end{aligned}
$$
therefore,

since we assume $N_{i}=t_{i} T$ and $N_{j}=t_{j} T$ so as $T \rightarrow \infty$ the first term in each side dominates the second term,

$$
\frac{-1}{\sqrt{2 \pi}} \frac{\alpha_{i}}{2 \sqrt{N_{i}}} \exp \left(\frac{-\alpha_{i}^{2} N_{i}}{2}\right)=\frac{1}{\sqrt{2 \pi}} \frac{\alpha_{j}}{2 \sqrt{N_{j}}} \exp \left(\frac{-\alpha_{j}^{2} N_{j}}{2}\right)
$$

Use the same argument in Case I and note that $\alpha_{i}<0$ and $\alpha_{j}>0$. We get

$$
\frac{N_{i}}{\sigma_{i}^{2} / \delta_{i}^{2}}=\frac{N_{j}}{\sigma_{j}^{2} / \delta_{j}^{2}}
$$

- Case III: $i, j \notin S_{m}$

Using (11), and by similar calculations, we get

$$
\frac{\alpha_{i}}{\sqrt{N_{i}}} \exp \left(\frac{-\alpha_{i}^{2} N_{i}}{2}\right)=\frac{\alpha_{j}}{\sqrt{N_{j}}} \exp \left(\frac{-\alpha_{j}^{2} N_{j}}{2}\right)
$$

Use the same argument in Case I, we get

$$
\frac{N_{i}}{\sigma_{i}^{2} / \delta_{i}^{2}}=\frac{N_{j}}{\sigma_{j}^{2} / \delta_{j}^{2}}
$$


Therefore for all cases by (12), (13) and (14), we have

$$
\frac{N_{i}}{\sigma_{i}^{2} / \delta_{i}^{2}}=\frac{N_{j}}{\sigma_{j}^{2} / \delta_{j}^{2}}
$$

Therefore, we have the following theorem

Given a total budget of simulation runs $T$ to be allocated to $k$ computing systems whose performance is depicted by random variables with means $Y\left(\theta_{1}\right), Y\left(\theta_{2}\right), \ldots, Y\left(\theta_{k}\right)$, and finite variances $\sigma_{1}^{2}, \sigma_{2}^{2}, \ldots, \sigma_{k}^{2}$ respectively, as $T \longrightarrow \infty$, the approximate probability of correct selection can be asymptotically maximized when

$$
\frac{N_{i}}{N_{j}}=\left(\frac{\sigma_{i} / \delta_{i}}{\sigma_{j} / \delta_{j}}\right)^{2}, \quad i, j \in\{1,2, \ldots, k\}
$$

where $N_{i}$ is the number of samples allocated to system $i, \delta_{i}=\bar{Y}_{i}-c$, and $\bar{Y}_{i}=\frac{1}{N_{i}} \sum_{k=1}^{N_{i}} Y_{i j}$, and $Y_{i j}$ is a sample from $Y_{i}$ for $j=1,2, \ldots, N_{i}, i=1,2, \ldots k$.

\subsection{Approximating the probability of correct selection}

In the previous section, we have seen how to distribute the available budget in order to maximize the probability of correct selection. Note that if we fix an index $s$ say, then we have

$$
\frac{N_{s}}{\sigma_{s}^{2} / \delta_{s}^{2}}=\frac{N_{j}}{\sigma_{j}^{2} / \delta_{j}^{2}} \quad \forall j=1, \ldots, k
$$

so we have

$$
N_{j}=\frac{\sigma_{j}^{2}}{\delta_{j}^{2}} \frac{\delta_{s}^{2}}{\sigma_{s}^{2}} N_{s}=\frac{\alpha_{s}^{2}}{\alpha_{j}^{2}} N_{s}
$$

recall that $\alpha_{j}=\delta_{j} / \sigma_{j}$. Therefore we have

$$
\alpha_{i}^{2} N_{i}=\alpha_{s}^{2} N_{s}, \forall i=1, \ldots k
$$

Since $\sum_{j \in \Theta} N_{j}=T$, we get

$$
\sum_{j \in \Theta} \frac{\alpha_{s}^{2}}{\alpha_{j}^{2}} N_{s}=T
$$

To facilitate the computation, let $D_{s}=\sum_{j \in \Theta} \frac{\alpha_{s}^{2}}{\alpha_{j}^{2}}$ and $D_{j}=\alpha_{s}^{2} / \alpha_{j}^{2}$ thus

$$
\begin{aligned}
& N_{s}=T / D_{s} \\
& N_{j}=D_{j} N_{s}, j \in \Theta, j \neq s
\end{aligned}
$$

Note that if $j \in S_{m}$, then $\alpha_{j}<0$ and if $j \notin S_{m}$, then $\alpha_{j}>0$. Therefore, plug (16) in (5), we can approximate $P(C S)$ as follows

$$
\begin{aligned}
P\left(C S^{*}\right) & =\prod_{i \in S_{m}}\left[1+\frac{1}{\sqrt{2 \pi}} \frac{1}{\sqrt{N_{i}} \alpha_{i}} \exp \left(\frac{-\alpha_{i}^{2} N_{i}}{2}\right)\right] \prod_{i \notin S_{m}}\left[1-\frac{1}{\sqrt{2 \pi}} \frac{1}{\sqrt{N_{i}} \alpha_{i}} \exp \left(\frac{-\alpha_{i}^{2} N_{i}}{2}\right)\right] \\
& =\left[1-\frac{1}{\sqrt{2 \pi}} \frac{1}{\sqrt{N_{s}}\left|\alpha_{s}\right|} \exp \left(\frac{-\alpha_{s}^{2} N_{s}}{2}\right)\right]^{k}
\end{aligned}
$$

To illustrate, we consider the following example.

To test the validity of formula (18) for approximating $P(C S)$, we implement it on a generic example consists of 20 systems each is normally distributed with mean $1+0.1 j, j=1, \ldots, 20$ and variance 1 . We assume that the correct selection is to select a set of 5 best solutions (where best means the lowest mean), of course the set $S_{m}=\{1.1,1.2,1.3,1.4,1.5\}$, we also let $c=1.55$ between the largest mean in $S_{m}$ and the smallest mean outside $S_{m}$. Table 1 includes the number of samples to be allocated to each system for $T=5,000$ simulation samples. Table 2 includes the approximated values of $P(C S)$ as a function of $T$, where $T$ ranges from 1,000 to 20,000 . 
Table 1. The approximation number of samples to be allocated to each system if $T=5000$ simulation runs

\begin{tabular}{cccc}
\hline Mean & $\alpha$ & $\alpha^{2}$ & $N_{j}$ \\
\hline 1.1 & -0.45 & 0.2025 & 26 \\
1.2 & -0.35 & 0.1225 & 43 \\
1.3 & -0.25 & 0.0625 & 83 \\
1.4 & -0.15 & 0.0225 & 231 \\
1.5 & -0.05 & 0.0025 & 2083 \\
1.6 & 0.05 & 0.0025 & 2083 \\
1.7 & 0.15 & 0.0225 & 231 \\
1.8 & 0.25 & 0.0625 & 83 \\
1.9 & 0.35 & 0.1225 & 43 \\
2 & 0.45 & 0.2025 & 26 \\
2.1 & 0.55 & 0.3025 & 17 \\
2.2 & 0.65 & 0.4225 & 12 \\
2.3 & 0.75 & 0.5625 & 9 \\
2.4 & 0.85 & 0.7225 & 7 \\
2.5 & 0.95 & 0.9025 & 6 \\
2.6 & 1.05 & 1.1025 & 5 \\
2.7 & 1.15 & 1.3225 & 4 \\
2.8 & 1.25 & 1.5625 & 3 \\
2.9 & 1.35 & 1.8225 & 3 \\
3 & 1.45 & 2.1025 & 2 \\
\hline
\end{tabular}

Table 2. The approximate values of $P(C S)$

\begin{tabular}{cccccccccc}
\hline$T$ & 1,000 & 2,000 & 4,000 & 6,000 & 8,000 & 10,000 & 12,000 & 15,000 & 20,000 \\
\hline$P(C S)$ & 0.0003 & 0.0513 & 0.4960 & 0.8191 & 0.9410 & 0.9810 & 0.9938 & 0.9988 & 0.9999 \\
\hline
\end{tabular}

It is clear that the systems with mean 1.5 and 1.6 are given more samples, in order to get a precise estimate of their means to take the correct decision. It is also clear from Table 2 that $P(C S)$ approaches 1 as the number of samples increased. The formula also implemented by alrefaei et al. [28] on larger example and indeed performs well.

Almomani and Alrefaei [21] presented an OO-OCBA $m$ algorithm for selecting the best subset for large size problems. Now we present a modification of the OO-OCBA $m$. The proposed modification of OO-OCBA $m$ procedure for selecting $m$ good enough systems:

- Step 0: Determine the number of initial simulation samples $t_{0} \geq 5$ and the number of samples to be distributed over the set of systems in each iteration, $\Delta$. Let $l=0$ where $l$ represents iteration index. Let $(1-\alpha) \%$ be the saught $P(C S *)$, and let $R$ be the maximum number of replications of the $O C B A_{m}$ inside each iteration and let $r=0$ and $T T=0$.

- Step 1: Select a set $G$ of size $g$ from the feasible solution set $\Theta$, let $N_{i}^{0}=t_{0}$ for all $i \in G$, and $N_{i}^{0}=0$ if $i \notin G, i \in \Theta$. Take random samples of $t_{0}$ observations $Y_{i j}\left(1 \leq j \leq t_{0}\right)$ for each system $i \in G$.

- Step 2: For each $i \in G$, compute the sample mean $\bar{Y}_{i}$ and the sample variance $s_{i}^{2}$ as; $\bar{Y}_{i}=\frac{1}{N_{i}^{r}} \sum_{j=1}^{N_{i}^{r}} Y_{i j}$ and $s_{i}=$ $\frac{1}{N_{i}^{r}-1} \sum_{j=1}^{N_{i}^{r}}\left(Y_{i j}-\bar{Y}_{i}\right)^{2}$.

- Step 3: Increase the computing budget by $\Delta$ and compute the new budget allocation $N_{i}^{r+1}, i \in G$ using (17).

- Step 4: Perform additional $\max \left\{0, N_{i}^{r+1}-N_{i}^{r}\right\}$ simulation samples for each system $i \in G$. Compute the new sample means and variances, let $r \leftarrow r+1$, if $r<R$ go to step 3 .

- Step 5: Let $S_{m}$ be the subset that contains the $m$ best mean systems. Let $N_{i}^{0}=N_{i}^{r}$ for all $i \in G$ and let $T T=$ $T T+\sum_{i \in G} N_{i}^{0}$. Compute the probability of correct selection $P(C S *)$ using Equation 18, if $P(C S *) \geq 1-\alpha \%$ then go to step 6, otherwise if $T T<T$, randomly select a subset $S_{c}$ of $g-m$ alternatives from $\Theta-G$ and replace the worst $g-m$ systems of the set $G$ with $S_{c}$. For $i \in S_{c}$, if $N_{i}^{0}=0$, let $N_{i}^{0}=t_{0}$, take random samples of $N_{i}^{0}$ observations $Y_{i j}\left(1 \leq j \leq N_{i}^{0}\right)$ and calculate the sample mean $\bar{Y}_{i}$ and sample variance $s_{i}^{2}$ for all $i \in G=S_{m} \cup S_{c}$. Let $l \longleftarrow l+1, r=0$, go to step 3 .

- Step 6: Select the set $S_{m}$ that contains the best systems in $G$.

\section{RESULTS AND DISCUSSION}

To illustrate the proposed algorithm, we implement it on solving inventory model under different parameter settings. Consider a stochastic inventory model with periodic review $(S, s)$. It is a single item under periodic review, 
full backlogging. The lead time is assumed to be uniform random variable between 0.5 and 1.0 period. The ordering cost includes a fixed set-up cost of $\$ 32$ per order and a $\$ 3$ per item, with inventory cost of $\$ 1$ per item per period. The shortage cost is $\$ 5$ per item per period. The time between demands is assumed to be i.i.d exponential random variable and the number of demand items in each demand is distributed as follows: $1,2,3$, and 4 , with probability $1 / 6,1 / 3,1 / 3$, and $1 / 6$, respectively.

We test 15-systems with the following policy; $\left(S_{i}, s_{i}\right)=\{(40,70),(50,100),(50,90),(60,130),(70,150),(90,140)$, $(50,200),(90,200),(60,90),(100,230),(50,190),(60,190),(70,100),(80,190),(100,130)\}$. First, we run simulation for each system to select the best of these systems, and we have the best as a follows $(40,70),(50,90)$, and $(60,90)$. Then, apply the approach using this policies to select the best, also we study the influence of the simulation factors, such that the initial sample size $t_{0}$, the increment in simulation samples $\Delta$, and the total budget used $\mathrm{T}$, in the performance of the proposed approach.

We perform the selection procedure that we develop on the given inventory example that we have discussed above. Algorithm 2.2 is codded using Java programming. For selecting the best $m$-system, we assume $m=3, \Delta=20$, and three choice of $t_{0}$ are considered; $t_{0}=5,10,20$. The outcome are shown in Figure 1. It is clear that the smaller the value of $t_{0}, t_{0}=5$ gives better performance than the larger size $t_{0}=10$ or 20 this is because we pend more samples on non competent solutions. Moreover, to get estimates of the mean and variance of each solution, we have to have non zer values of $t_{0}$. It is also clear that the procedure converges to unity for all choices of $t_{0}$ which suggest that the algorithm is robust. Now we perform the selection procedure on the above example with different values of $\Delta ; \Delta=10,20,50$, and $t_{0}=20$. Figure 2 shows the results of selecting the best $m$-system, we choose $m=3, t_{0}=20$. When the computing budget equal 750 the $P(C S)$ that we have is $0.966,0.9,0.933$ when $\Delta=10,20,50$, respectively. So the better choice here is when $\Delta=10$. In conclusion, the smaller the values of the initial sample size $t_{0}$, the better solution we get. The number of samples to be performed and distributed over the alternatives $\Delta$ is better to be moderate.

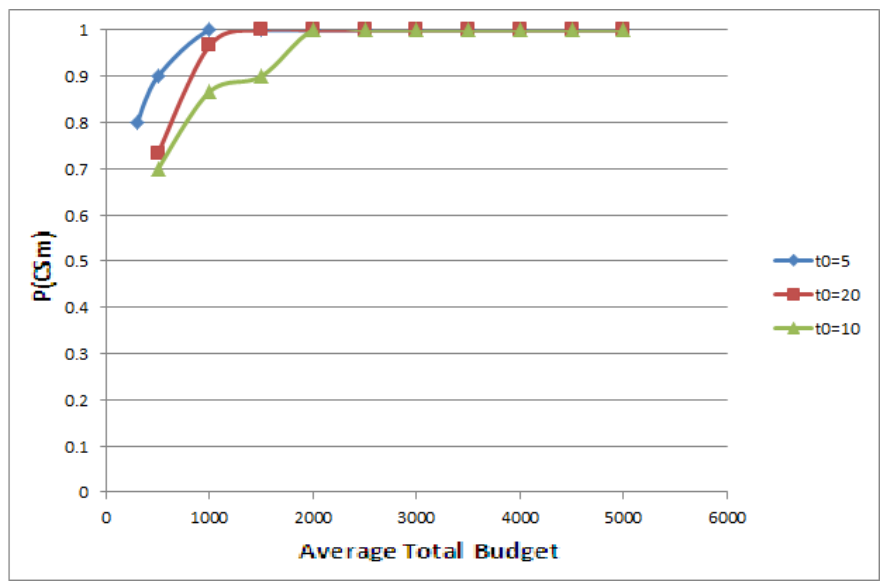

Figure 1. The effect of $t_{0}$ over 10 replications when selecting the best $m$-system

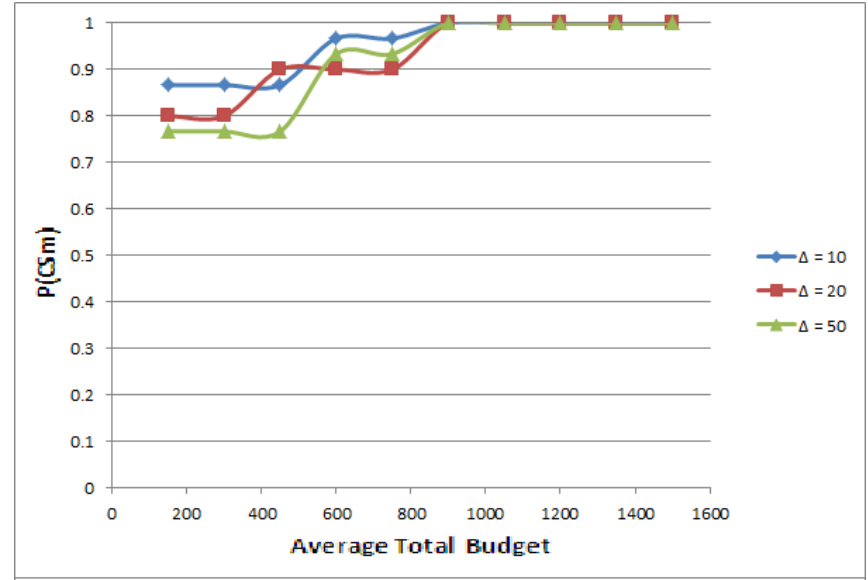

Figure 2. The effect of $\Delta$ over 10 replications when selecting the best $m$-system 


\section{CONCLUSION}

In this paper, we have derived an approximation of the probability of correct selection when $\mathrm{OCBA}_{m}$ is used to select the best $m$ systems. Then, we have presented a sequential approach for selecting a set of good enough systems, from a large set of alternative systems. The proposed approach consists of two stages. In the first stage, we use the $O O$ procedure to select a subset $G$ randomly from the search space that contains the best simulated system with high probability. In the second stage, we use the $O C B A_{m}$ procedure to select the best $m$ subset of $G$ which is chosen by $O O$ procedure. The proposed selection approach is applied on monotone increasing mean example and solving inventory model with different parameter setting. The numerical results show that the algorithm selects a set of best systems very fast.

\section{ACKNOWLEDGEMENT}

This research work was supported in part by Jordan University of Science and Technology, under Research Grant number: 20170268

\section{REFERENCES}

[1] C. H. Chen, E. Yücesan and S. E. Chick, "Simulation budget allocation for further enhancing the efficiency of ordinal optimization,” Discrete Events Dynamic Systems, vol. 10, no. 3, pp. 251-270, 2000.

[2] H. Xiao and L. H. Lee, "Simulation optimization using genetic algorithms with optimal computing budget allocation," SIMULATION, vol. 90, no. 10, pp. 1146-1157, 2014

[3] S. Zhang, L. H. Lee, E. P. Chew, J. Xu and C. Chen, "A Simulation Budget Allocation Procedure for Enhancing the Efficiency of Optimal Subset Selection,” IEEE Transactions on Automatic Control, vol. 61, no. 1, pp. 62-75, 2016, doi: 10.1109/TAC.2015.2423832.

[4] H. Xiao, H. Chen and L. H. Lee, "Optimal computing budget allocation for ranking the top designs with stochastic constraints," in 2017 Winter Simulation Conference (WSC) Las Vegas, NV, 2017, pp. 2218-2224, doi: 10.1109/WSC.2017.8247953.

[5] S. Zhang, J. Xu, L. H. Lee, E. P. Chew, W. P. Wong and C. Chen, "Optimal Computing Budget Allocation for Particle Swarm Optimization in Stochastic Optimization," IEEE Transactions on Evolutionary Computation vol. 21, no. 2, pp. 206-219, 2017.

[6] Y. Zhu, G. Pedrielli, and L. H. Lee, "TD-OCBA: Optimal Computing Budget Allocation and Time Dilation for Simulation Optimization of Manufacturing Systems," IISE Transactions vol. 51, no. 3, pp. 219-231, 2019.

[7] J. Kennedy and Eberhart, "Particle Swarm Optimization," Proceedings of IEEE International Conference on Neural Networks IV, 1995, pp. 1942-1948.

[8] A Abubaker, A Baharum, M Alrefaei, "Multi-objective particle swarm optimization and simulated annealing in practice," Applied Mathematical Sciences, vol. 10, no. 42, pp. 2087-2103, 2016

[9] V. Manusov, P. Matrenin and N. Khasanzoda, "Swarm algorithms in dynamic optimization problem of reactive power compensation units control," International Journal of Electrical and Computer Engineering (IJECE), vol. 9, no. 5, pp. 3967-3974, 2019, doi: 10.11591/ijece.v9i5.pp3967-3974.

[10] A. Yoganandini and G. Anitha, "A modified particle swarm optimization algorithm to enhance MPPT in the PV array," International Journal of Electrical and Computer Engineering (IJECE), vol. 10, no. 5, pp. 5001-5008, 2020, doi: 10.11591/ijece.v10i5.pp5001-5008.

[11] M. Abd Rahman, B. Ismail, K. Naidu, and M. Rahmat, "Review on population-based metaheuristic search techniques for optimal power flow," International Journal of Electrical and Computer Engineering (IJECE), vol. 15, no. 1, pp. 373-381, 2019, doi: 10.11591/ijeecs.v15.11.pp373-381.

[12] Y. Liu, G. Pedrielli, H. Li, L. H. Lee, C. Chen, and J. F. Shortle, "Optimal computing budget allocation for stochastic Nk problem in the power grid system," IEEE Transactions on Reliability, vol. 68, no. 3, pp. 778-789, 2019, doi: 10.1109/TR.2019.2913741.

[13] D. Cao, Y. Sun and H. Guo, "Optimizing Maintenance Policies based on Discrete Event Simulation and the OCBA Mechanism," Reliability and Maintainability Symposium, January, 2013.

[14] Y. C. Ho, R. S. Sreenivas, and P, Vakili, "Ordinal optimization of DEDS," Discrete Events Dynamic Systems, vol. 2, no. 1, pp. 61-88, 1992.

[15] E. J. Chen, "Using ordinal optimization approach to improve efficiency of selection procedures," Discrete Event Dynamic Systems: Theory and Applications, vol. 14, pp. 153-170, 2004.

[16] A. M. Hasan, and S. M. Rafaat, "Optimized formation control of multi-agent system using PSO algorithm," Indonesian Journal of Electrical Engineering and Computer Science (IJEECS), vol. 20, no. 3, pp. 1591-1600, 2020.

[17] E. Asmae, K. Loubna, B. Bachir and Z. Izeddine., "Meta-heuristic techniques for optimal design of analog and digital filter," Indonesian Journal of Electrical Engineering and Computer Science, vol. 19, no. 2, pp. 669-679, 2020.

[18] S. Gao, L. H. Lee, C. Chen and L. Shi, "A Sequential Budget Allocation Framework for Simulation Optimization," IEEE Transactions on Automation Science and Engineering, vol. 14, no. 2, pp. 1185-1194, 2017. 
[19] M. H. Alrefaei and A. J. Alawneh, "Selecting the best stochastic system for large scale problems in discrete event dynamic systems," Mathematics and Computers in Simulation, vol. 64, pp. 237-245, 2004.

[20] M. H. Almomani and R. Abdul Rahman, "Selecting a good stochastic system for the large number of alternatives," Communications in Statistics-Simulation and Computation, vol. 41, pp. 222-237, 2012.

[21] M. H. Almomani and M. H. Alrefaei, "Ordinal optimization with optimal computing budget for selecting an optimal subset," Asia-Pacific Journal of Operational Research, vol. 33, doi: 10.1142/S0217595916500093, 2016.

[22] M. Al-Salem, M. H. Almomani, M. H. Alrefaei and A. Diabat, "On the optimal computing budget allocation problem for large scale simulation optimization," Simulation Modelling Practice and Theory, vol. 71, pp. 149-159, 2017, doi: 10.1016/j.simpat.2016.05.004.

[23] M. H. Almomani, M. H. Alrefaei and S. A. Mansour, "A combined statistical selection procedure measured by expected opportunity cost," Arabian Journal for Science and Engineering, vol. 43, no. 6, pp. 3163-3171, 2018, doi: 10.1007/s13369-017-2865-8.

[24] M. H. Almomani, M. H. Alrefaei and S. A. Mansour, "A method for selecting the best performance systems," International Journal of Pure and Applied Mathematics, vol. 120, no. 2, pp. 191-202, 2018.

[25] M. H. Almomani, A. Baharum and M. H. Alrefaei, "A selection approach for solving the buffer allocation problem," International Journal of the Physical Sciences, vol. 7, no. 3, pp. 413-422, 2012.

[26] C. H. Chen, D. He, M. Fu and L. H. Lee, "Efficient simulation budget allocation for selecting an optimal subset," Informs Journal on Computing, vol. 20, no. 4, pp. 579-595, 2008.

[27] S. Ross, "A first Course in Probability," $4^{\text {th }}$ ed. Macmillan, 1994.

[28] M. H. Alrefaei, M. H. Almomani and S. A. Alhadi, "Asymptotic approximation of the probability of correctly selecting the best systems," AIP Conference Proceedings, vol. 1991, no. 1, 2018, Art. No. 020022, doi: 10.1063/1.5047895. 